\title{
A novel HDAC inhibitor suppresses extracellular vesicle VEGF-C-mediated lymphangiogenesis and pancreatic cancer early dissemination
}

\section{Chu-An Wang}

National Cheng Kung University College of Medicine

Chien-Feng Li

Chi Mei Foundation Hospital: Chi Mei Medical Center

Rho-Chi Huang

National Cheng Kung University College of Medicine

Yo-Hua Li

National Cheng Kung University College of Medicine

Jing-Ping Liou

Taipei Medical College: Taipei Medical University

Shaw-Jenq Tsai ( $\sim$ seantsai@mail.ncku.edu.tw )

National Cheng Kung University College of Medicine

\section{Research}

Keywords: DUSP2, HDAC inhibitor, PDAC, VEGF-C

Posted Date: September 8th, 2020

DOl: https://doi.org/10.21203/rs.3.rs-70831/v1

License: (1) This work is licensed under a Creative Commons Attribution 4.0 International License. Read Full License 


\section{Abstract \\ Background}

Pancreatic ductal adenocarcinoma (PDAC) is a highly aggressive cancer characterized by early dissemination and poor drug response. Loss-of-function of dual specificity phosphatase 2 (DUSP2), a critical regulator of MAPKs signaling, is highly associated with cancer malignancies. Therefore, it may provide new therapeutic strategy if the actions of DUSP2 can be restored.

\section{Methods}

The tumor suppressor role of DUSP2 was demonstrated via DUSP2 re-expression in the orthotopic mouse model of pancreatic cancer and knockout of Dusp 2 in the pancreas by transgenic mouse model. Immunohistochemical staining and histology analysis was performed to evaluate tumor development and progression. Bioinformatic analysis was utilized to identify potential drug which mimics DUSP2 reexpression. Pancreatic cancer cell survival, migration ability, and the expression and function of extracellular vesicle (EV) associated vascular endothelial growth factor C (VEGF-C) was measured. The effect of the novel HDAC inhibitor on pancreatic cancer progression was evalulated by orthotopic mouse model.

\section{Results}

Forced expression of DUSP2 abrogated tumor formation and loss of Dusp2 facilitated Kras-driven PDAC formation. Increased HDAC1 expression was found in PDAC and inhibition of HDAC showed similar gene profile as Kras knockdown and DUSP2 re-expression. Treatment with B390 inhibited growth and migration abilities of PDAC cells, decreased EV-associated VEGF-C expression, and suppressed lymphatic endothelial cell proliferation. In vivo, B390 not only suppressed tumor growth by increasing tumor cell death, it also inhibited lymphangiogenesis and lymphovascular invasion.

\section{Conclusions}

Our data provide the proof-of-concept evidence to demonstrate the potential of using novel HDAC inhibitor in PDAC treatment which alleviates loss-of-DUSP2-mediated pathological processes.

\section{Background}

Pancreatic ductal adenocarcinoma (PDAC) accounts for more than $90 \%$ of pancreatic malignancies and is notorious for the poor prognosis with a less than $6 \%$ five-year survival rate. PDAC is rarely diagnosed in pre-metastatic state and lymphovascular invasion (LVI) is frequently observed in PDAC patients (1). As in other types of solid tumors, increased LVI and lymph node metastasis correlates with unfavorable 
outcome in PDAC (2). LVI can be the consequence that tumor cells stimulate the formation of blood/lymphatic vessels via secreting factors and gain of invasion ability. Since PDAC is highly metastatic, it is devastating to understand the mechanism of early metastasis and identify means to prevent it in order to improve patient survival.

KRAS mutation occurs in more than $90 \%$ of PDAC. Inhibition of MAPKs/ERK suppresses the growth of Kras driven pancreatic cancer growth in transgenic mouse model $(3,4)$, indicating the necessity of MAPKs/ERK signaling. Dual specificity phosphatases (DUSPs) are protein phosphatases that can simultaneously dephosphorylate serine/threonine and tyrosine residues of the same substrate. DUSP2 belongs to the subgroup of MAPK phosphatase (MKPs) that antagonize the activation of MAPK cascades, mainly via negatively regulates ERK activation. As ERK is the convergent point of multiple signaling pathways, dysregulation of DUSP2 will lead to uncontrolled cellular responses and is associated with disease progression (5). Downregulation of DUSP2 has been found in leukemia and many solid tumors $(6,7)$. It has been demonstrated that DUSP2 expression is suppressed by hypoxia and downregulation of DUSP2 promotes drug resistance and cancer stemness $(6,8)$. In pancreatic cancer, loss of DUSP2 was observed at the early stage of pancreatic intraepithelial neoplasia (PanIN), and DUSP2 downregulation promotes LVI in the orthotopic mouse model (9). Considering the critical roles of DUSP2, identification of means to restore its function may be an alternative way to alleviate PDAC malignancy.

In this study, we aimed to investigate whether re-expression of DUSP2 is sufficient to suppress PDAC progression. More importantly, to identify novel inhibitor that can mimic DUSP2's function. Our results indicate that HDAC inhibitor functions like DUSP2 and can be used to inhibit tumor growth and prevent lymphovascular invasion in mouse model of PDAC.

\section{Methods}

\section{Cell culture}

Pancreatic ductal adenocarcinoma cell line MIA PaCa-2 were maintained in RPMI 1640 complete medium containing $10 \%$ fetal bovine serum (FBS), glutamine, sodium pyruvate, and antibiotics for prevention of bacteria, fungi, and mycoplasma. HPDE-E6E7 was initially maintained in keratinocyte-serum free medium (50 mg/ml bovine pituitary extract) and gradually cultivated in RPMI 1640 complete medium. LECs were purchased (PromoCell) and cultured in endothelial cell growth medium MV2 with supplement (PromoCell). KPC cell line was isolated from a pancreatic tumor developed in KPC transgenic mouse model as reported (10) and maintained in RPMI 1640 complete medium. Cell lines were maintained at $37{ }^{\circ} \mathrm{C}, 5 \% \mathrm{CO}_{2}$ humidified incubator. MIA PaCa-2 and HPDE-E6E7 have been authenticated.

\section{Proliferation and migration assay}

MIA PaCa-2 cells were plated on 24-well plate and transfected with GFP or DUSP2-GFP plasmids. After 48 hours, MTT was added and crystal was dissolved in DSMO, OD590nm was measured and quantified. 
Isolated EV from control and B390 treated MIA PaCa-2 was resuspended in PBS and treated LECs for 48 hours, LECs were fixed and stained for Ki67 and DAPI. MIA PaCa-2 cells were suspended in serum-free RPMI media and placed in transwell chamber with a pore size of $8.0 \mu \mathrm{m}$ (PIEP12R48, Millipore). 10\% FBS RPMI culture media was used as chemoattractant. After 16 hours of incubation, migrated cells were fixed and stained with crystal violet. Cell numbers were counted and analyzed by ImageJ cell counter.

\section{Extracellular vesicles isolation and analysis:}

MIA PaCa-2 cells were plated on the first day and medium was changed for serum-free RPMI medium containing ITS-M (Insulin-Transferrin-Selenium Mixture, Genedirex) for one more day. Conditioned medium (CM) was first under centrifugation at $500 \mathrm{~g}$ for $10 \mathrm{~min}$ to remove cell debris and supernatant was further under centrifugation $16000 \mathrm{~g}$ for $30 \mathrm{~min}$. Processed CM were sent to the Center for Micro/Nano Science and Technology at National Chung Kung University for nanoparticle tracking analysis (NTA). EVs were extracted from the cell culture medium by ExoQuick exosome precipitation solution (SBI system Biosciences) according to the manufacturer's protocol.

\section{Western blotting and antibodies}

Whole cell lysates were collected by RIPA buffer and subjected to Western blotting as described previously (6). Antibodies against DUSP2 (Santa Cruz, sc-32776), phosphor-p44/42 MAPK (cell signaling, \#4370), p44/42 MAPK (cell signaling, \#4696), VEGF-C (GeneTex, GTX113574), CD9 (Proteintech, 602321), HSP70 (Santa cruz, sc-32239), GAPDH (GeneTex, GTX100118) were used.

\section{Immunohistochemistry (IHC)}

Tumors from animals were fixed in $4 \%$ paraformaldehyde, embedded in paraffin, and cut into $5-\mu \mathrm{m}$ sections and sections were stained with H\&E or IHC. Primary antibodies against Lyve-1 (Angiobio, 11034) and CD31 (abcam, ab28364) were used for immunohistochemistry staining of lymphangiogenesis and angiogenesis. Cleaved caspase 3 (cell signaling, 9661T) was used for the staining of apoptosis. HDAC-1 (cell signaling, \#5356 s) was used for staining of pancreas and tumor from mice.

\section{Animal studies:}

KC (LSL-Kras ${ }^{\text {G12D; }}$ Pdx-1-Cre ) and KPC (LSL-KrasG12D; TP53R172H; Pdx-1-Cre) transgenic mouse model were provided by Dr. Po-Hsien Huang, National Cheng Kung University. Conditional knockout of Dusp2 in the pancreas is referred to KDC mice (LSL-Kras ${ }^{\mathrm{G} 12 \mathrm{D}}$, Dusp2F/F, Pdx-1-Cre). CRISPR/Cas9 system was used for replacing endogenous mouse dusp2 as described (9). Mice were sacrificed at the age of 6 months and pancreases were harvested for IHC analysis.

Male SCID mice (6- to 8-weeks of age) were used for pancreas orthotopic injection. $1 \times 10^{6} \mathrm{MIA} \mathrm{PaCa-2}$ cells were suspended in $100 \mu \mathrm{l}$ of RPMI/matrigel mixture and injected into the pancreas of mice. For drug experiment, mice were randomly assigned to experimental or control groups after two weeks of 
inoculation. In the treatment group, mice received vehicle or B390 $(12.5 \mathrm{mg} / \mathrm{kg})$ by oral gavage with flexible feeding tubes, five days/week for two consecutive weeks (8). Mice were sacrificed three days after the last treatment and tumors were excised.

\section{Statistical analysis}

GraphPad Prism 5.00 was used for statistical analysis. Two-tailed Student's t-test and one-way ANOVA with Tukey posttests were employed for comparing two or three groups, respectively. Error bars represent standard error of the mean from independent experiments. Asterisks denote significant difference from control group *, $\mathrm{P}<0.05 ; * \star, \mathrm{P}<0.01 ; * \star \star, \mathrm{P}<0.001$.

\section{Results}

Expression of DUSP2 inhibits pancreatic cancer tumor formation

Our previous study demonstrated that DUSP2 is downregulated in PDAC (9); therefore, we aimed to investigate whether re-expression of DUSP2 can diminish tumor burden. MIA PaCa-2 cells, which expressed very low level of DUSP2, were transfected with plasmids containg GFP or DUSP2-GFP. Exogenously overexpression of DUSP2 decreased basal level of ERK activation in MIA PaCa-2 cells (Fig. 1A) and suppressed cell growth (Fig. 1B). To determine the effect of DUSP2 on tumor formation in vivo, control and DUSP2 expressing MIA Paca-2 cells were orthotopically injected into mouse pancreas and tumor growth was followed by luciferase imaging. Tumor formation was abrogated when DUSP2 was expressed (Fig. 1C). Indeed, when the animals were sacrificed, no tumor was observed in the DUSP2expressing group (Fig. 1C). Because there is no tumor developed in the DUSP2 overexpressing group, we can not evaluate how DUSP2 inhibited tumor growth. Therefore, another pancreatic orthotopic tumor model was generated by injecting control or DUSP2 knockdown (KD) PANC-1 cells. Immunohistochemical staining showed knckdown of DUSP2 increased Ki67-positive cells and markedly reduced number of apoptotic cells (Fig. 1D). These results indicate that DUSP2 function as a tumor suppressor and loss of DUSP2 promotes proliferation and survival of tumor cells during PDAC tumorigenesis.

Loss of Dusp2 promotes Kras driven PanIN progression in transgenic mouse model

Activating mutation of KRAS occurs in more than $90 \%$ of PDAC; however, mice carry Kras mutation in the pancreas develop pre-invasive PanIN but seldom progresses to invasive cancer $(11,12)$. Therefore, we aimed to evaluate the effect of Dusp2 loss in the background of Kras mutation. For this purpose, KDC mice (Pdx-1Cre, LSL-Kras ${ }^{\mathrm{G12D} /+}$, Dusp $\left.2^{\mathrm{f} / \mathrm{fl}}\right)$ were generated (Fig. 2A). Pancreas of KDC mice started to show histological abnormalities at 2 months of age (Fig. 2B). However, to evaluate the additional effect of Dusp2 loss, we compared the histology of pancreas from wild type, KC (Pdx-1Cre, LSL-Kras $\left.{ }^{\mathrm{G} 12 \mathrm{D} /+}\right)$, and KDC mice at 6 months of age. Pancreas of KC mice showd regions of acinar to ductual metaplasia (ADM), which was considered as the pre-neoplastic lesions of PDAC (13). PanINs were also observed in 6 months old KC pancreas (Fig. 2C, c-d). Of note, KDC mice not only showed more severe ADM and PanIN phenotypes but alo developed carcinoma surrounded by dense stroma in pancreas (Fig. 2C, e-f). Together, 
these results indicate that loss of Dusp2 speed up the development and progression of Kras-induced precancerous neoplasia.

Restoration of DUSP2-mediated functions by novel histone deacetylase inhibitor

Due to the difficulty in restoration of a tumor suppressor protein in reality, we aimed to identify molecules which may exert similar functions. By cross-referencing our previously published microarray dataset (8) with connectivity map (14), we identified a list of inhibitors that function like DUSP2 (Fig. 3A). Next, the CLUE Plateform (COMMAND) was utilized to search the mechanism of action of these inhibitors and identified that HDAC inhibitor functions similarly to KRAS knockdown (Fig. 3B), an indications of DUSP2 reactivation. Moreover, a public dataset contains transcriptome profiling of two class I/II HDAC inhibitors (sodium butyrate and Trichostatin A) (15) was utilized to cross with our own dataset of DUSP2 overexpression. 44 up-regulated genes and 21 down-regulated genes in both HDAC inhibitor datasets show significant fold change in DUSP2 dataset (Fig. 3C). Interestingly, DUSP2 was found up-regulated in both HDAC inhibitors treatment after 12 and 48 hours, implying HDAC inhibitor may exert similar tumor suppressive functions via reinforcement of DUSP2 expression. As the expression of HDACs in PDAC is largely uninvestigated, we analyzed microarray datasets from Oncomine and identified that the expression of some HDACs, especially HDAC1, is significantly increased in multiple datasets (Fig. 3D). We further performed immunohistochemistry on mouse pancreatic tumor developed in KPC transgenic mouse model (LSL-Kras ${ }^{G 12 D}$; TP53R ${ }^{172 H}$; Pdx-1-Cre), which has been demonstrated to resemble features of human PDAC (16). As compared to normal epithelial ducts (littermate control), increased HDAC1 can be detected in PanINs and carcinoma (Fig. 3E). These results suggest that HDAC1 may participate in the progression from normal duct to neoplasia.

The effects of novel HDAC inhibitors (B369 and B390) had recently been demonstrated in colon cancer which abolished hypoxia mediated cancer stemness (8). Thus, we first determined the dose response of normal and cancer cells to B369 and B390. Normal human pancreatic ductal epithelial cell line, HPDEE6E7, show no response to both B369 and B390 (Fig. 4A) while the growth of MIA Paca-2 was significantly inhibited after 48 hours treatment, speficially by B390 (Fig. 4B). Importantly, treatment of B369 and B390 increased the expression of DUSP2 in MIA Paca-2 cells (Fig. 4C), indicating novel HDAC inhibitors selectively kill cancer cells via re-expression of DUSP2. We also evalulated the effect of B390 on primary cancer cells isolated from pancreatic tumor developed in KPC mice, which is also sensitive to B390 induced death similar to MIA PaCa-2 cells (Fig. 4D). Next, migration ability, another attribute that DUSP2 regulates in pancreatic cancer cells was also measured. Pre-treatment of MIA Paca-2 cells with B390 for 24 hours significantly diminished migration ability compared to control (Fig. 4E).

Since we have recently demonstrated that DUSP2 knockdown promotes extracellular vesicles (EV)-VEGFC secretion and lymphangiogenesis (9), we thus investigated the effect of B390 on these processes. Reduced levels of EV-VEGF-C was observed (Fig. 4F) and further study revealed that B390 inhibited VEGFC expression at the transcriptional level (Fig. 4G), thus reducing its loading to EVs. Furthermore, in vitro assay showed that the proliferation of LECs was significantly inhibited when treated with EVs isolated 
from B390-treated MIA PaCa-2 cells (Fig. 4H and 4I). Together, B390 shows inhibitory effects similar to DUSP2 expression in pancreatic cancer cells.

Inhibition of tumor progression by B390 in orthotopic mouse model of pancreatic cancer

To determine the in vivo effect of B390, MIA PaCa-2 cells were injected into the pancreas of mouse and animals received vehicle or B390 for 3 consecutive weeks. Treatment with B390 decreased the size of tumors measured by IVIS imaging (Fig. 5A). We further performed histology analysis on the tumors. Multinodular tumors were observed in the control group while B390-treated tumors are smaller and with necrosis (Fig. 5B-C). In addition, enhanced cell death was observed in B390-treated tumors as stained by antbodies against cleaved caspase-3 ( an apoptotic marker) (Fig. 5D).

Since loss of DUSP2 promotes tumor-associated lymphangiogenesis, we thus determined the levels of angiogenesis and lymphangiogenesis in control and B390 treated mice. As shown, both angiogenesis (CD31) and lymphangiogenesis (Lyve-1) are significantly inhibited in B390 treated group (Fig. 6A-B). Histology examination showed two out of five mice displayed vascular invasion (Fig. 6C and Fig. 6 Da-b) and one out of five shows tumor cells seeding in the fat tissue in the control group (Fig. 6Dc). In contrast, no invasion was observed in B390 treated tumors (right, $n=7$ ). Together, these results suggest that novel HDAC inhibitors decrease VEGF-C expression and exert multiple effects in inhibiting cancer progression, partially mimicking the effect of DUSP2 re-expression.

\section{Discussion}

Pancreatic cancer is a very aggressive type of tumor that is often diagnosed with locally advanced stage or metastatic spread. Survival duration for patients with non-resectable tumors is only 4-8 months and chemotherapy shows limited effects (17). While gemcitabine has been used to treat metastatic PDAC, resistance develops rapidly. Therefore, seeking an effective therapy is the high priority to improve patients' survival. In this study, we demonstrate DUSP2 acts as a tumor suppressor that exogenous expression is sufficient to restrain tumor formation. Furthermore, we identified that novel HDAC inhibitor, B390 functions like DUSP2. In addition to abolishing cancer cell growth, B390 inhibits EV-associated VEGF-C expression and lymphatic invasion, by which DUSP2 regulates. Orthotopic mouse model demonstrates B390 not only promotes tumor apoptosis but also inhibits tumor spreading into the surrounding tissue. Together, our study suggests the anti-tumor function of the novel HDAC inhibitor in recapitulating the action of DUSP2 in suppressing PDAC progression.

DUSP2 belongs to a subclass of dual specificity phosphatase and is inducibly expressed in the immune system (18). Loss of DUSP2 expression correlates with cancer malignancies and constitutive ERK activation $(6-8,19)$. Recently, we demonstrated that loss-of-DUSP2 promotes pancreatic cancer early dissemination, likely through lymphovasculature (9). In this study, we show that re-expression of DUSP2 is sufficient to prevent tumor formation in the orthotopic mouse model of pancreatic cancer. Also, we found that knockout of Dusp2 facilitates the progression of Kras-mediated PDAC development, 
suggesting the critical gatekeeper role of DUSP2. Therefore, we reason that identifying a way to restore the expression or function of DUSP2 may be able to inhibit PDAC progression.

By using bioinformatic analysis and cross-referencing with online databases, we found that DUSP2 functions similar to several inihibitors. No surprise that action of mitogen-activated protein kinase (MEK) inhibitors was identified with the top score, reflecting that DUSP2 overexpression works similar as MEK inhibition. However, since KRAS mutation tumors show intrinsic resistance to MEK inhibitor (20); therefore, we seeked for different drugs. Interestingly, we found that HDAC inhibitors exert similar functions like DUSP2, which prompted us to consider whether administration of HDAC inhibitor can restore DUSP2 expression or mimic DUSP2's function in suppressing cancer progression. Since overexpression of HDACs has been found in different types of cancer and is associated with tumor grade, prognosis, and chemoresistance (21); therefore, targeting HDAC has always been considered as an anticancer approach. Indeed, there are many recruiting or active clinical trials using HDAC inhibitors or in combination with other drugs. In general, HDAC inhibitors exert anti-cancer effects via reactivating scilenced tumor suppressor genes by epigenetic regulation or inducing cancer cell death. For example, trichostatin A inhibits pancreatic cancer cell growth via inducing cell-cycle arrest and apoptosis (22), or induces the re-expression of tumor suppressor gene such as transforming growth factor beta type II receptor (23). In this study, we identify unrevealed functions of HDAC inhibitor, which advances our understandings about the actions of HDAC inhibitor's anti-cancer mechanism.

Apoptosis is one of the major mechanisms that HDAC inhibitors selectively kill cancer cells. However, there are still some caveats of how HDAC inhibitors promote apoptosis. In this study, we observed the massive activation of caspase-3 in B390-treated tumors, which is similar to DUSP2 overexpression phenotype (Fig. 1 and reference (6)). As DUSP2 is frequently suppressed by hypoxic stress in solid cancers (6), loss-of-DUSP2 warrants the cancer cells to grow without triggering the proliferation-induced apoptosis switch. The inactivation of apoptosis plays a central role in cancer development. By orthotopic mouse model, we observed that loss of DUSP2 showed less apoptosis in the tumor regions. These results indicate that DUSP2 may be responsible for stress induced cell death and thus loss of function promotes survival of tumor cells and facilitates cancer progression. The anti-cancer function of HDAC inhibitors may be mediated through re-activation of DUSP2 or DUSP2-regulated downstream effectors. By using bioinformatic analysis of our previous published microarray data and online databases, we found HDAC inhibitors and DUSP2 regulate many common biological processes. Since cancer cells treated with B390 showed increased expression of DUSP2, implying the anti-cancer effects of novel HDAC inhibitors may be due to the restoration of DUSP2 expression. However, the detail mechanism of how loss of DUSP2 overlaps with HDAC1/2-mediated functions warrants further invesitagtion.

The most exciting finding in this study is that we identified a novel mechanism of HDAC inhibitor and evaluated rarely investigated but highly critical pathophysiological processes in cancer progression including EV function and lymphangiogenesis. Our previous data showed that knockdown of DUSP2 increased EV-associated VEGF-C in PDAC, which facilitated lymphangiogenesis and ultimately results in pancreatic cancer cells early dissemination (9). Since previous study showed that treatment with HDAC 
inhibitors rescued hypoxia mediated DUSP2 downregulation in colon cancer, we hypothesized that B390 may abrogate loss-of-DUSP2-induced EV-VEGF-C secretion and lymphangiogenesis. Our results suggest that treatment with B390 significantly inhibited the mRNA expression of VEGF-C, thus resulting in the reduction of EV-VEGFC in pancreatic cancer cells. Importantly, B390 suppressed tumor cells invaded into lymphovasculature and the surrounding tissue, a phenomenon particularly critical for PDAC malignancy. These data reveal the previously uncharacterized novel effects of HDAC inhibitor in regulating cancer early dissemination and malignancy.

\section{Conclusions}

Our results reveal the tumor suppressor function of DUSP2 in pancreatic cancer and unravel a novel mechanism of HDAC inhibitor in mouse model of PDAC. B390 not only induces apoptosis of cancer cells but also inhibits tumor invasion through lymphavascular system. Since dissemination through lymaphtic system represents a novel and previously not well-characterized route for cancer cells, especially in PDAC, our study shed light on the potential use of B390 or similar HDAC inhibitors as anti-cancer drugs in inhibiting pancreatic cancer progression.

\section{Abbreviations}

PDAC

Pancreatic ductal adenocarcinoma

DUSP2

dual specificity phosphatase 2

HDAC

histone deacetylase

EV

extracellular vesicles

VEGF-C

vascular endothelial growth factor $\mathrm{C}$

\section{Declarations}

\section{Ethics approval:}

Experimental procedures of animal studies were approved by the Institutional Animal Care and Use Committee at the National Chung Kung University.

\section{Availability of data and materials:}

The datasets during and/or analysed during the current study available from the corresponding author on reasonable request. 
Consent for publication:

Not applicable.

Competing interests:

The authors declare that they have no competing interests.

\section{Fudning}

This work was supported by grants from Ministry of Science and Technology (106-2321-B-006 - 022MY3, MOST 108-2321-B-006-006 -) and National Health Research Institute (NHRI-EX105-10516BI).

\section{Authors' Contributions:}

Chu-An Wang designed and performed experiments, analyzed data, and wrote the initial draft of the manuscript. Chien-Feng Li examined the pathology of mouse and human sections. Rho-Chi Huang performed in vitro drug response experiments and Yo-Hua Li perfomed one mouse orthotopic experiment. Jing-Ping Liou synthesized and provided the HDAC inhibitors. SJ Tsai conceived the project and provided the critical review of the manuscript.

\section{Acknowledgement:}

We thank Taiwan Bioinformatics Institute Core Facility for assistance on using Oncomine (National Core Facility Program for Biotechnology, MOST 108-2319-B-400-001).

We thank Yi-Shang Yeh, Yi-Chen Tang, and Yi-Jou Chung for the technical support in immunohistochemistry staining and animal experiments. We thank Yen-Yu Lai for bioinformatics analysis. We thank the technical services provided by the "Bioimaging Core Facility of the National Core Facility for Biopharmaceuticals, Ministry of Science and Technology, Taiwan". We thank for Dr. Po-Hsien Huang, Department of Biochemistry and molecular biology, National Cheng Kung University for the KC transgenic mice.

\section{Disclosure of potential conflicts of interest}

No potential conflicts of interest were disclosed.

\section{References}


1. Fink DM, Steele MM, Hollingsworth MA. The lymphatic system and pancreatic cancer. Cancer letters. 2016;381(1):217-36.

2. Wang Z, Wu J, Li G, Zhang X, Tong M, Wu Z, et al. Lymphangiogenesis and biological behavior in pancreatic carcinoma and other pancreatic tumors. Mol Med Rep. 2012;5(4):959-63.

3. Hayes TK, Neel NF, Hu C, Gautam P, Chenard M, Long B, et al. Long-Term ERK Inhibition in KRASMutant Pancreatic Cancer Is Associated with MYC Degradation and Senescence-like Growth Suppression. Cancer cell. 2016;29(1):75-89.

4. Collins MA, Yan W, Sebolt-Leopold JS, Pasca di Magliano M. MAPK signaling is required for dedifferentiation of acinar cells and development of pancreatic intraepithelial neoplasia in mice. Gastroenterology. 2014;146(3):822-34. e7.

5. Wei W, Jiao Y, Postlethwaite A, Stuart JM, Wang Y, Sun D, et al. Dual-specificity phosphatases 2: surprising positive effect at the molecular level and a potential biomarker of diseases. Genes Immun. 2013;14(1):1-6.

6. Lin SC, Chien CW, Lee JC, Yeh YC, Hsu KF, Lai YY, et al. Suppression of dual-specificity phosphatase-2 by hypoxia increases chemoresistance and malignancy in human cancer cells. J Clin Invest. 2011;121(5):1905-16.

7. Kim SC, Hahn JS, Min YH, Yoo NC, Ko YW, Lee WJ. Constitutive activation of extracellular signalregulated kinase in human acute leukemias: combined role of activation of MEK, hyperexpression of extracellular signal-regulated kinase, and downregulation of a phosphatase, PAC1. Blood. 1999;93(11):3893-9.

8. Hou PC, Li YH, Lin SC, Lin SC, Lee JC, Lin BW, et al. Hypoxia-Induced Downregulation of DUSP-2 Phosphatase Drives Colon Cancer Stemness. Cancer research. 2017;77(16):4305-16.

9. Wang CA, Chang IH, Hou PC, Tai YJ, Li WN, Hsu PL, et al. DUSP2 regulates extracellular vesicle-VEGFC secretion and pancreatic cancer early dissemination. J Extracell Vesicles. 2020;9(1):1746529.

10. Torres MP, Rachagani S, Souchek JJ, Mallya K, Johansson SL, Batra SK. Novel pancreatic cancer cell lines derived from genetically engineered mouse models of spontaneous pancreatic adenocarcinoma: applications in diagnosis and therapy. PloS one. 2013;8(11):e80580.

11. Ariston Gabriel AN, Jiao Q, Yvette U, Yang X, Al-Ameri SA, Du L, et al. Differences between KC and KPC pancreatic ductal adenocarcinoma mice models, in terms of their modeling biology and their clinical relevance. Pancreatology. 2020;20(1):79-88.

12. Gopinathan A, Morton JP, Jodrell DI, Sansom OJ. GEMMs as preclinical models for testing pancreatic cancer therapies. Dis Model Mech. 2015;8(10):1185-200.

13. Chuvin N, Vincent DF, Pommier RM, Alcaraz LB, Gout J, Caligaris C, et al. Acinar-to-Ductal Metaplasia Induced by Transforming Growth Factor Beta Facilitates KRAS(G12D)-driven Pancreatic Tumorigenesis. Cell Mol Gastroenterol Hepatol. 2017;4(2):263-82.

14. Subramanian A, Narayan R, Corsello SM, Peck DD, Natoli TE, Lu X, et al. A Next Generation Connectivity Map: L1000 Platform and the First 1,000,000 Profiles. Cell. 2017;171(6):1437-52 e17. 
15. Mackmull MT, Iskar M, Parca L, Singer S, Bork P, Ori A, et al. Histone Deacetylase Inhibitors (HDACi) Cause the Selective Depletion of Bromodomain Containing Proteins (BCPs). Mol Cell Proteomics. 2015;14(5):1350-60.

16. Olive KP, Tuveson DA. The use of targeted mouse models for preclinical testing of novel cancer therapeutics. Clinical cancer research: an official journal of the American Association for Cancer Research. 2006;12(18):5277-87.

17. Krska Z, Svab J, Hoskovec D, Ulrych J. Pancreatic Cancer Diagnostics and Treatment-Current State. Prague Med Rep. 2015;116(4):253-67.

18. Lang R, Hammer M, Mages J. DUSP meet immunology: dual specificity MAPK phosphatases in control of the inflammatory response. J Immunol. 2006;177(11):7497-504.

19. Lin SC, Hsiao KY, Chang N, Hou PC, Tsai SJ. Loss of dual-specificity phosphatase-2 promotes angiogenesis and metastasis via up-regulation of interleukin-8 in colon cancer. J Pathol. 2017;241(5):638-48.

20. Sun C, Hobor S, Bertotti A, Zecchin D, Huang S, Galimi F, et al. Intrinsic resistance to MEK inhibition in KRAS mutant lung and colon cancer through transcriptional induction of ERBB3. Cell reports. 2014;7(1):86-93.

21. Eckschlager T, Plch J, Stiborova M, Hrabeta J. Histone Deacetylase Inhibitors as Anticancer Drugs. Int J Mol Sci. 2017;18(7):1414.

22. Damaskos C, Garmpis N, Karatzas T, Nikolidakis L, Kostakis ID, Garmpi A, et al. Histone Deacetylase (HDAC) Inhibitors: Current Evidence for Therapeutic Activities in Pancreatic Cancer. Anticancer research. 2015;35(6):3129-35.

23. Huang W, Zhao S, Ammanamanchi S, Brattain M, Venkatasubbarao K, Freeman JW. Trichostatin A induces transforming growth factor beta type II receptor promoter activity and acetylation of Sp1 by recruitment of PCAF/p300 to a Sp1.NF-Y complex. J Biol Chem. 2005;280(11):10047-54.

\section{Figures}


A

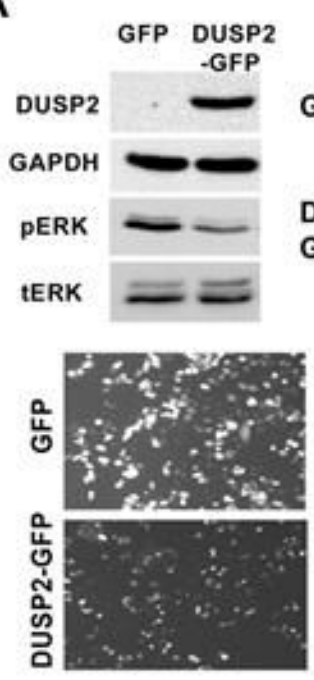

D
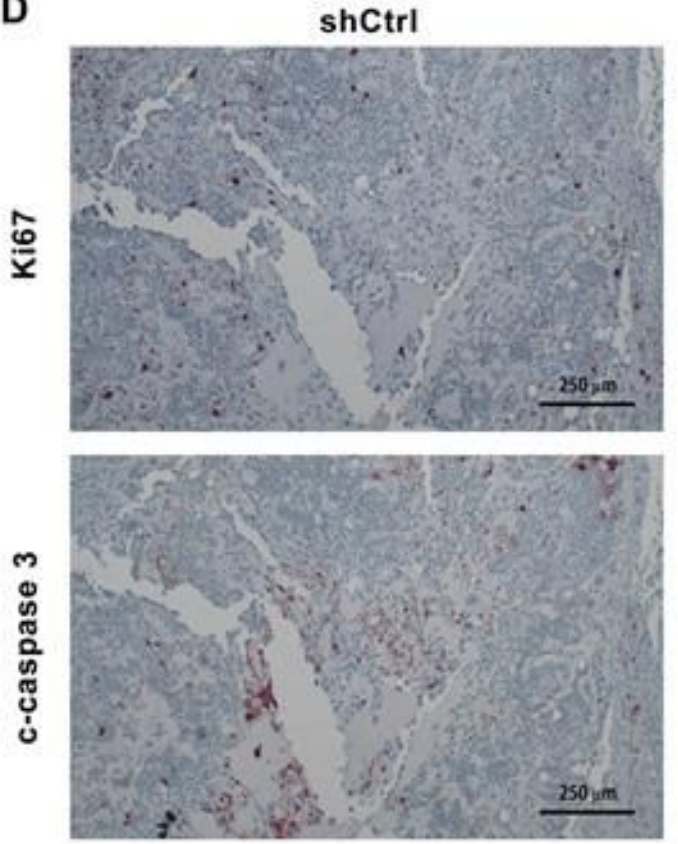

C
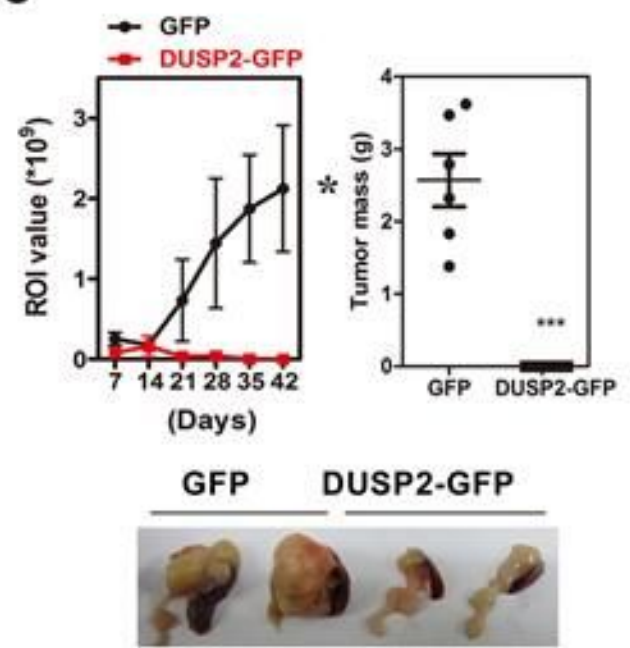

shDUSP2

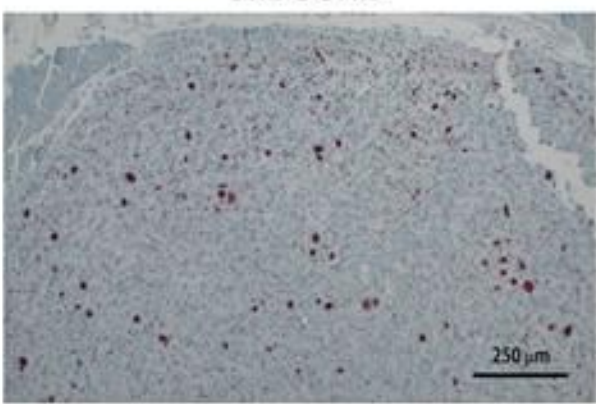

$250 \mu \mathrm{m}$

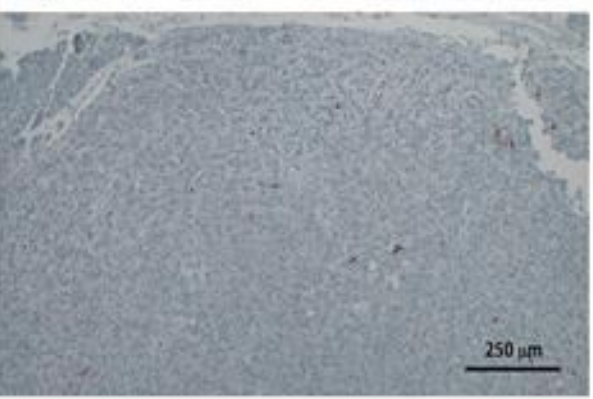

\section{Figure 1}

Exogenous expression of DUSP2 inhibits pancreatic tumor formation. (A) Western blotting for the expression of DUSP2 and ERK1/2 in MIA PaCa-2 cells transfected with GFP or DUSP2-GFP expression vector. GAPDH is the internal control for whole cell lysate (upper). Representative images shows the fluroscent signal of GFP in GFP or DUSP2-GFP transfected MIA PaCa-2 cells (bottom). (B) Representative images show crystal violet-stained cells growing on 24-well plates for 48 hours. Equal numbers of MIA PaCa-2 cells were plated on 24-well plates and transfected with GFP or DUSP2-GFP expression vectors. This experiment was repeated by MTT assay with similar result (bottom). (C) Growth curve (upper left), tumor mass (upper right) and representative image (bottom) show forced expression of DUSP2 abolished tumor growth in mice pancreas. GFP or DUSP2-GFP MIA PaCa-2 cells were labeled with luciferase and injected into mouse pancreas ( $\mathrm{n}=6$ mice per group). Tumor mass was followed by IVIS imaging. 42 days 
after inoculation, mice were sacrificed, tumors and pancreases were collected. (D) Immunohistochemistry of Ki67 and cleaved caspase 3 in control (shCtrl) and DUSP2 knockdown (shDUSP2) PANC-1 tumors.

A
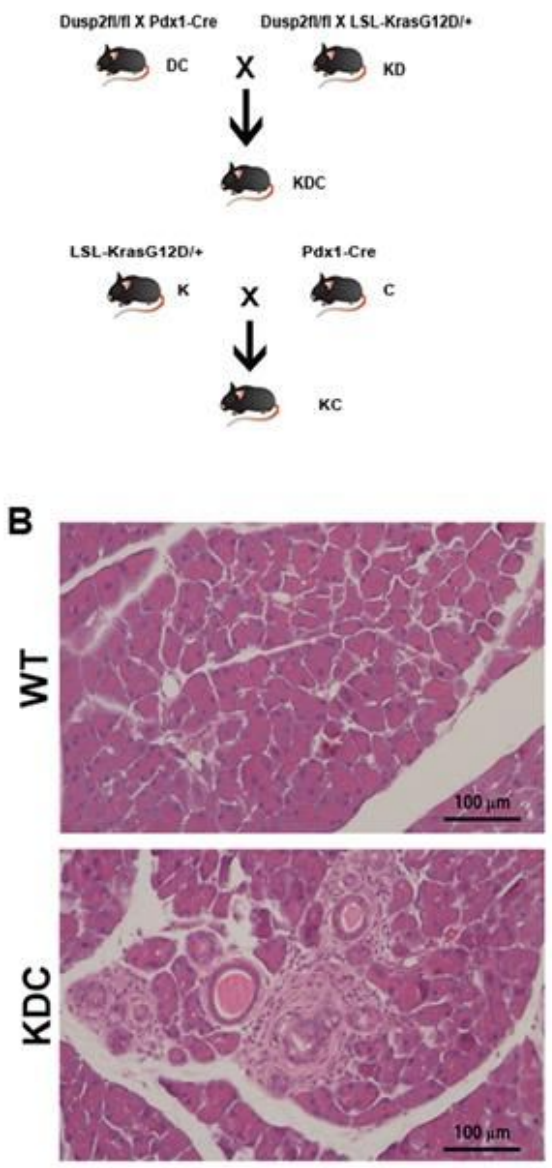

C
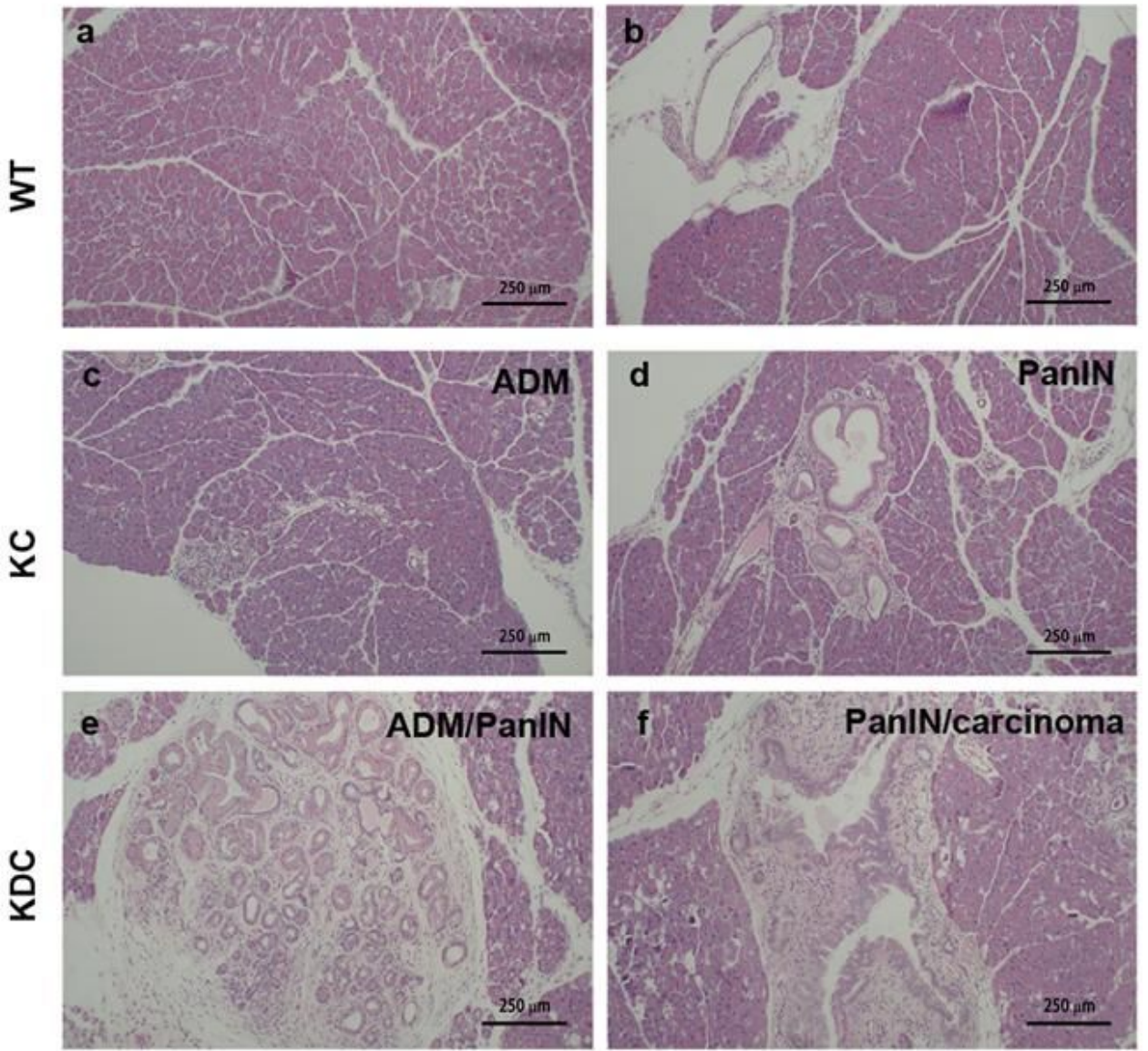

\section{Figure 2}

Loss of Dusp2 in the pancreas facilitates the progression of kras-driven PanIN progression. (A) Schmetic diagram of breeding KC and KDC transgenic mouse model. (B) Histology of pancreas from 2 months old KDC mice and wild type littermate. (C) Haematoxylin and eosin (H\&E) staining of pancreas in six months old wild type (a-b), KC mice (c-d) and KDC mice (e-f). Normal pancreatic ducts (b) shows single layer of epithelial cells. Pancreas of KC shows acinar to ductual metaplasia (ADM) and PanINs while pancreas of KDC display larger area of abnormalities, including ADM, PanINs and carcinoma. Early PanIN display papillary or micropapillary projections. Advanced PanIN has epithelial cells budding into the lumen and necrosis in the lumen. Carcinoma displays nuclear abnormalities and glands embedded in tumor stroma. 
A

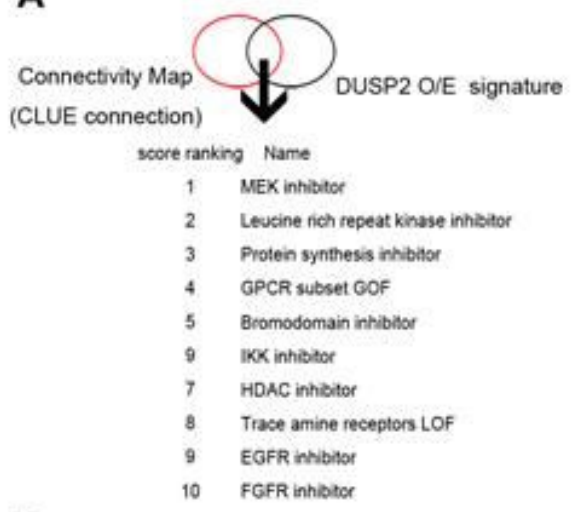

B

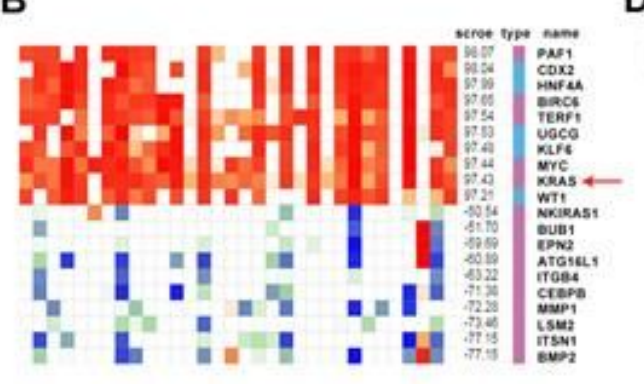

D
C

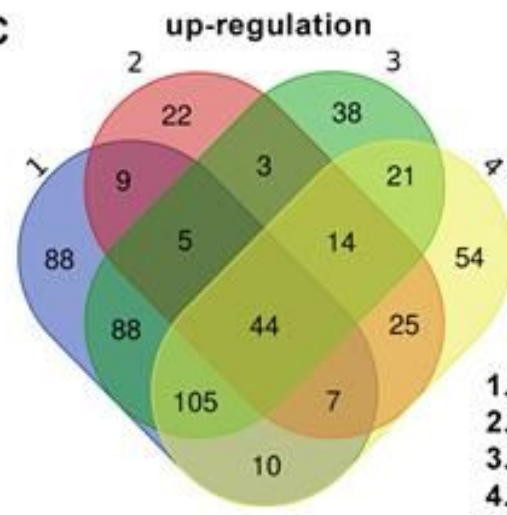

DUSP2 O/E

$\begin{array}{rrr}\text { Median Rank } & \text { p-Value } & \text { Gene } \\ 2771.0 & 6.826-7 & \text { HDAC1 } \\ 2803.0 & 0.064 & \text { HDACB } \\ 3251.5 & 0.053 & \text { HDAC9 } \\ 5184.0 & 0.146 & \text { HDAC2 } \\ 5690.0 & 0.327 & \text { HDNC7 } \\ 7868.5 & 0.288 & \text { HDNC6 } \\ 8421.0 & 0.318 & \text { HDAC4 } \\ 9261.0 & 0.331 & \text { HDACS } \\ 9702.0 & 0.473 & \text { HDAC3 } \\ 12746.5 & 0.792 & \text { HDAC11 } \\ 15564.0 & 0.889 & \text { HDAC10 }\end{array}$

1. TSA $12 \mathrm{hr}$

2. TSA $48 \mathrm{hr}$

3. Butyrate $12 \mathrm{hr}$

4. Butyrate $48 \mathrm{hr}$

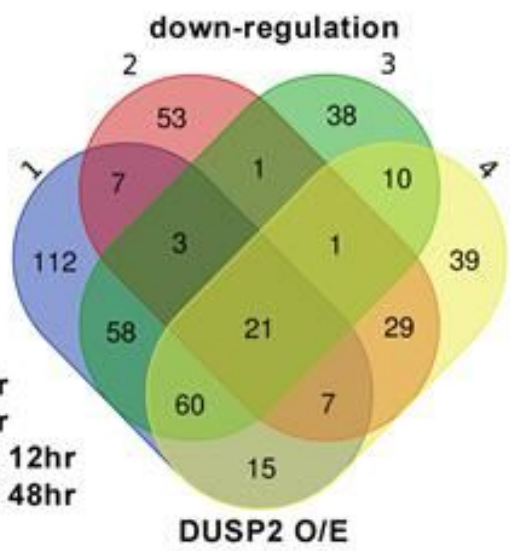

DUSP2 O/E

E
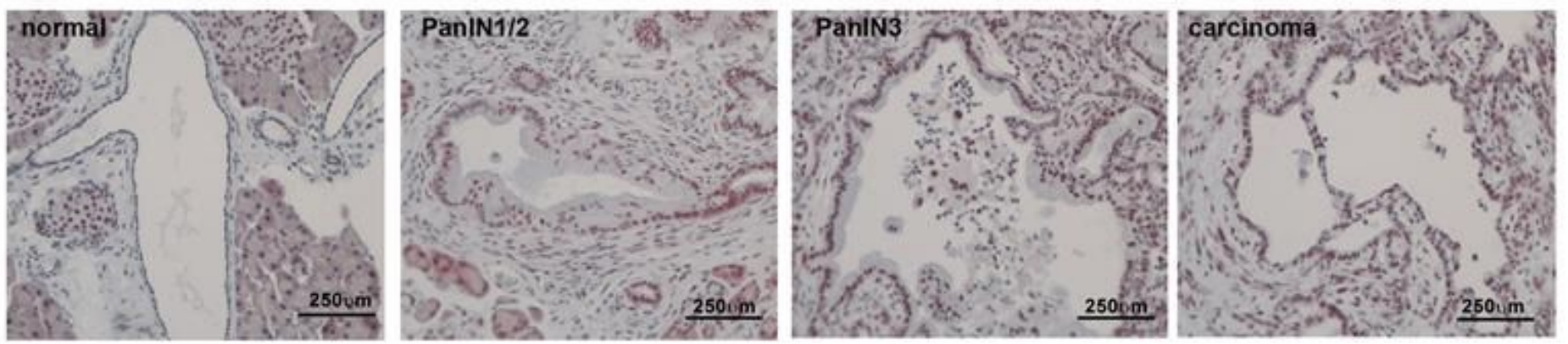

\section{Figure 3}

Reexpression of DUSP2 shows overlapping gene signature as that in cells treated with HDAC inhibitor. (A) Gene signatures of DUSP2 overexpression (GSE66656) was crossed reference with the connectivity map (CMAP, Broad institute) to identify action of drugs. (B) The CLUE Plateform (COMMAND) was utilized to search the mechanism of action of HDAC inhibitors. As indicated, knocdown of KRAS, shows similarity to profiles of HDAC inhibitors by genetic perturbations. (C) Comparative transcriptome profiling of HDAC inhibitors and DUSP2 expression. Microarray analysis of gene expression in TSA and sodium butyrate treated cells. Two time points (12 and 48 hours) were included for analysis. Overlapping gene list (up or down) was further crossed with DUSP2 expression dataset. (D) HDACs expression in human pancreatic tumors compared to normal pancreas in seven independent datasets from Oncomine. Oncomine ${ }^{\mathrm{TM}}$ (Compendia Bioscience, Ann Arbor, MI) was used for analysis and visualization. (E) 
Immunohistochemistry of HDAC1 in normal pancreas from wild type mouse and pancreatic tumors from KPC mouse.
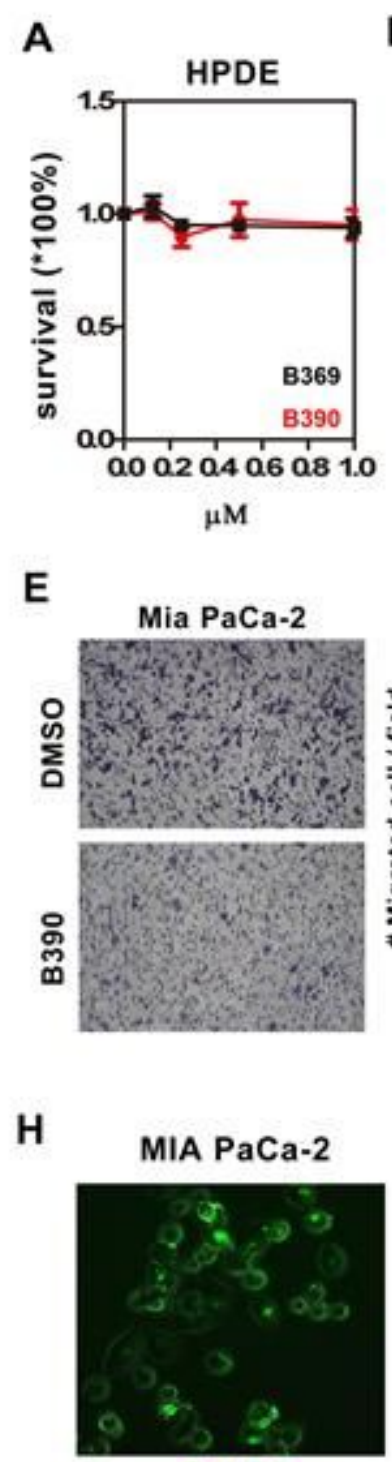

PKH67
B

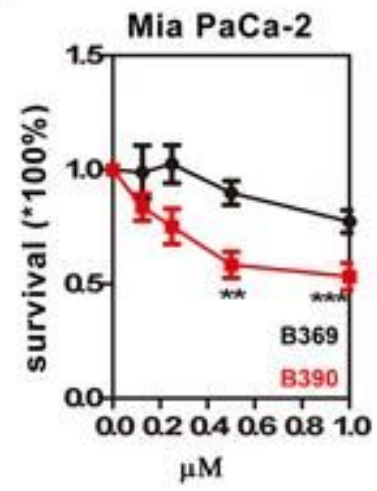

$\mathbf{F}$
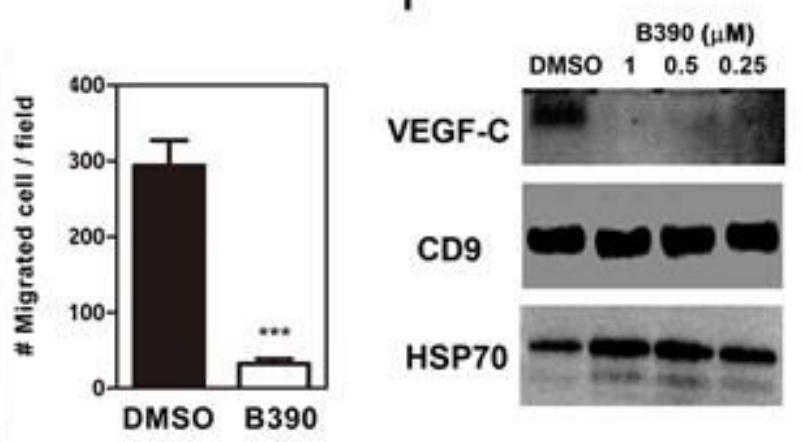

I

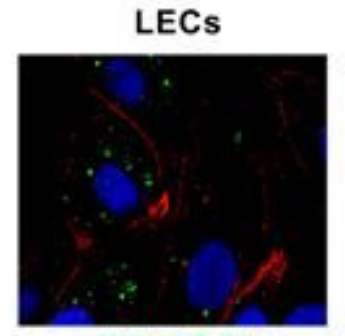

PKH67-EV

Phalloidin/DAPI
C
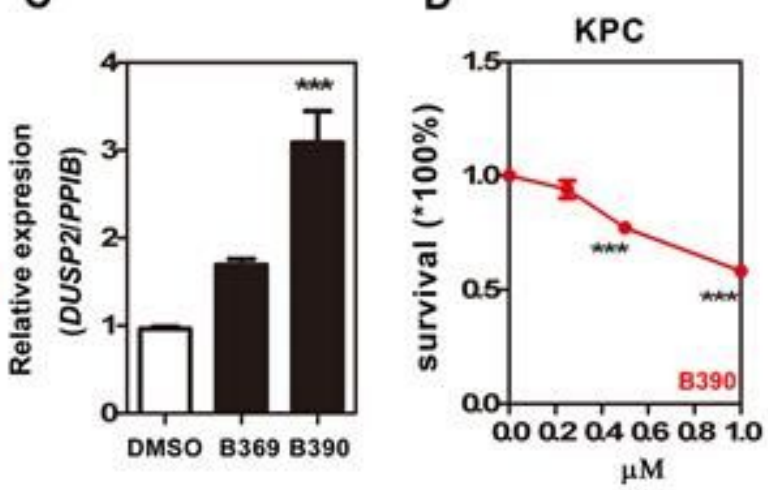

G
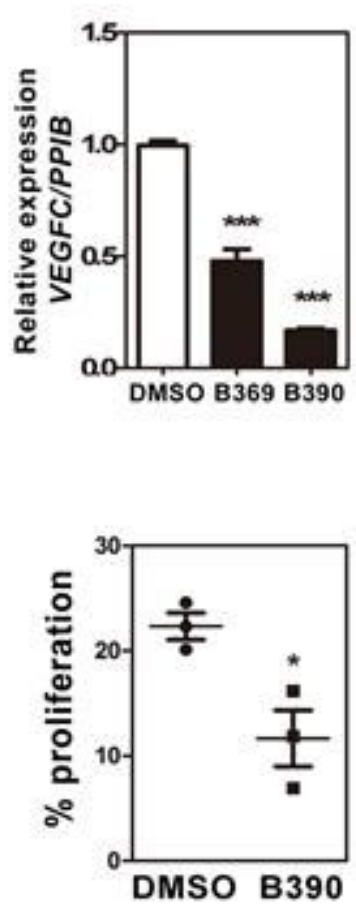

Figure 4

B390 inhibits pancreatic cancer migration, EV-VEGF-C expression, and proliferation of lymphatic endothelial cells. (A-B) Cell viability of HPDE-E6E7 and MIA PaCa-2 treated with DMSO and different doses of B369 or B390 for 48 hours. Cell viability was assessed by MTT assay and the value of O.D. reading was normalized back to the value of control (DMSO). The results represent the means of three independent experiments. (C) RT-qPCR of DUSP2 expression in MIA PaCa-2 cells treated with B369 and B390 $(1 \mu \mathrm{M})$ for 24 hours. CYCLOPHILIN B (PPIB) was used as internal control. (D) Cell viability of KPC cells treated with DMSO and different doses of B390 for 48 hours. (E) Representative images (left) and 
quantitative result (right, $\mathrm{n}=3$ ) show migration assay in MIA PaCa-2 cells pre-treated with DMSO or B390 $(1 \mu \mathrm{M})$ for 24 hours. (F) Serum free conditioned medium (CM) of control and B390 treated MIA PaCa-2 cells was collected and EV was isolated by Exoquick-TC. Western blotting was performed to detect VEGFC expression. CD9 and HSP70 were used as markers for EV. (G) RT-qPCR of VEGFC in MIA PaCa-2 cells treated with B369 and B390 $(1 \mu \mathrm{M})$ for 24 hours. CYCLOPHILIN B (PPIB) was used as internal control. (H) A selected confocal image shows LECs uptake EV from MIA PaCa-2 cells. MIA PaCa-2 cells were labeled with fluorescence dye PKH67 (left) and CM was collected. EV was isolated from CM to treat lymphatic endothelial cells (LECs) for 6 hours (right). (I) Representative images (left) and quantitative result (right, $\mathrm{n}=3$ ) show proliferation (Ki67 staining) of LECs treated with EV from control or B390 treated MIA PaCa-2 cells for 48 hours. ${ }^{*} \mathrm{P}<0.05$; $* * \mathrm{P}<0.01$;*** $\mathrm{P}<0.001$.

A
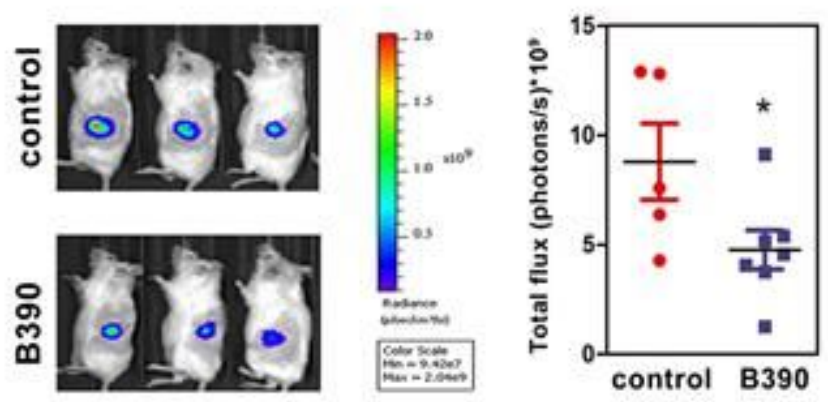

B

DMSO

B390
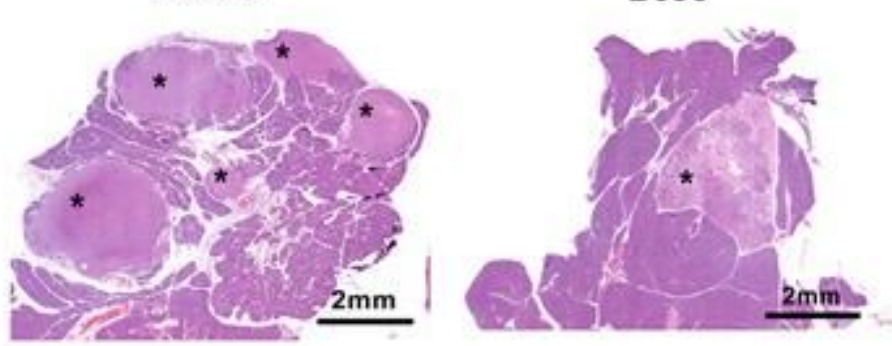

C

DMSO

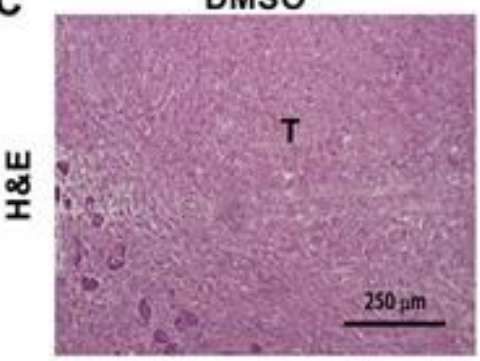

B390

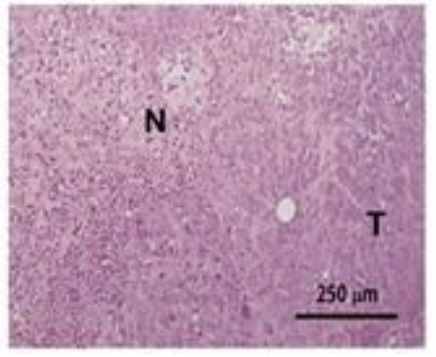

D

DMSO

B390
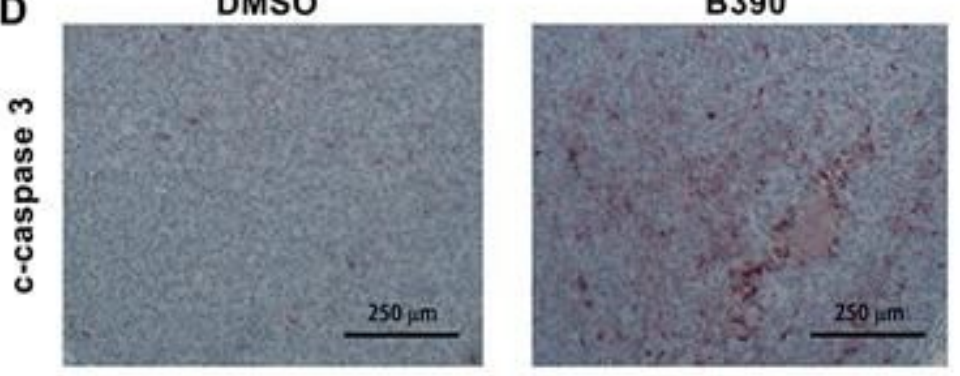

Figure 5 
Treatment of B390 reduces pancreatic tumor mass and promotes tumor cell death. (A) Bioluminescent imaging of SCID mice injected of MIA PaCa-2 in the pancreas and received control and B390 treatment (left). False color logarithmic scale represents the intensity of the bioluminescent signal in photons. Quantification of the total flux of the bioluminescent signal in control and B390 treated mice (right). (B) Representative image of whole pancreas tissues stained by hematoxylin and eosin (H\&E). Asterisk indicates tumor regions in control and B390 treated tumors. (C) Representative image of H\&E stain in control and B390 treated tumors. Tumor necrosis $(\mathrm{N})$ was observed in B390 treated tumor. Purple hematoxiphilic signal indicate viable tumor tissues ( $T$ ). (D) Representative immunohistochemistry staining for cleaved caspase 3 as indicator of apoptosis. As compared to control tumor, B390 treated tumors have increased signal for apoptosis.

A

Control
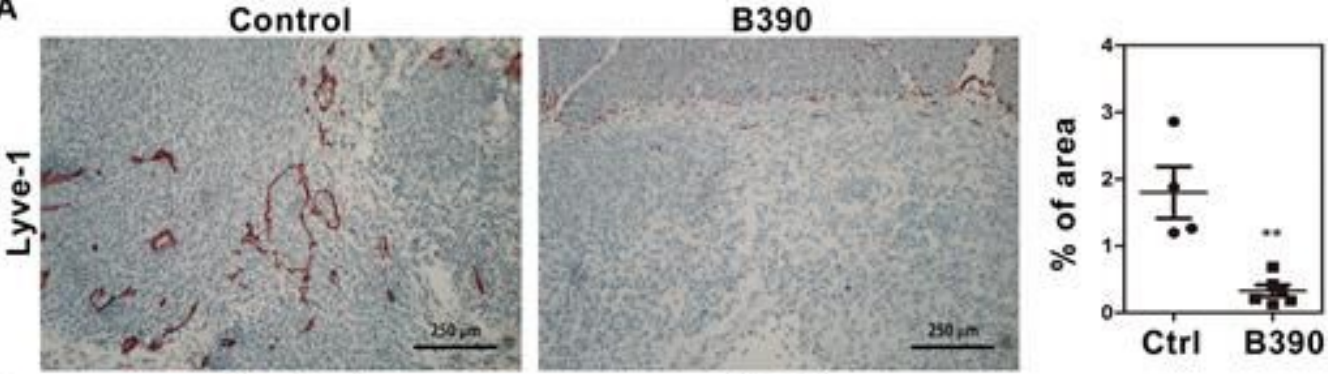

B

Control

B390
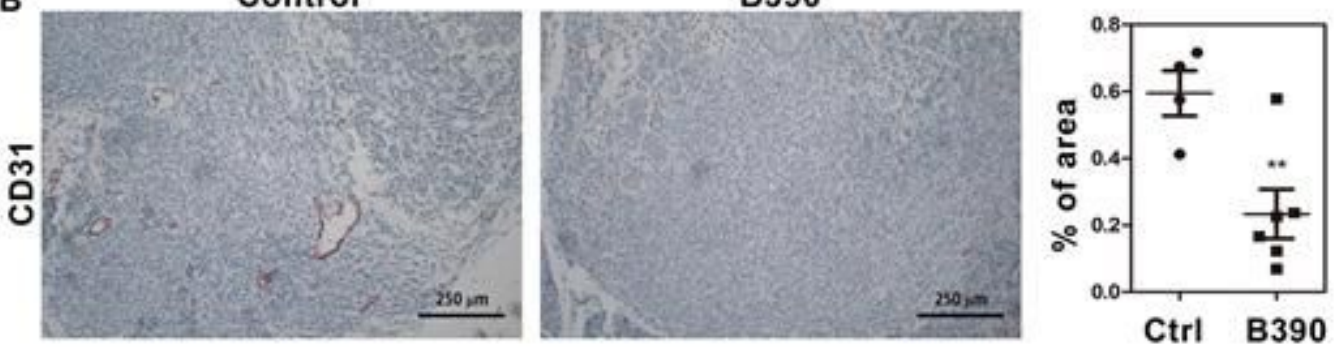

C

Control

B390
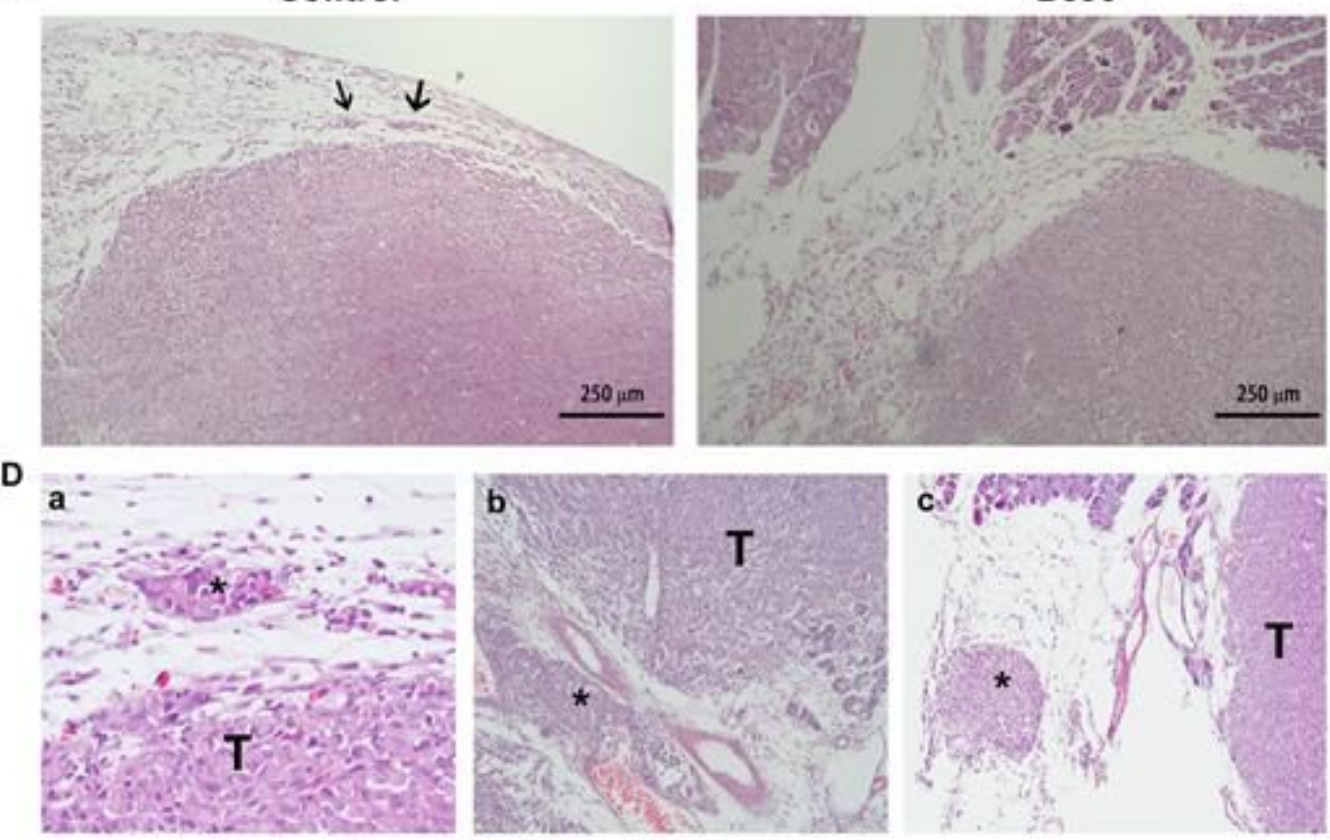

Figure 6 
B390 treatment reduces tumor-associated lymphangiogenesis and tumor invasion. (A-B) Representative images show the decrease of lymphatic (Lyve-1) and blood vessels (CD31) in B390 treated MIA PaCa-2 tumors (left). Quantification of Lyve-1 and CD31 positive are in four tumors from control and six tumors from B390 treated grouped (right).(C) Representative image of tumor and surrounding area in control and B390 treated group by H\&E stain. Arrows indicate vascular invasion. (D) Representative images of vascular invasion ( $a$ and $b$ ) and fat tissue seeding $(c)$ in MIA PaCa-2 control tumors by H\&E stain. T denotes tumor area and asterisk denotes invaded tumor cells. 